US Army Corps

of Engineers

Prepared for the U.S. Army Corps of Engineers, Portland District,

under an Interagency Agreement with the U.S. Department of Energy

Contract DE-AC05-76RL01830

\title{
Juvenile Salmon \\ Acoustic Telemetry System Transmitter Downsize Assessment
}

\author{
TJ Carlson \\ MJ Myjak
}

April 2010

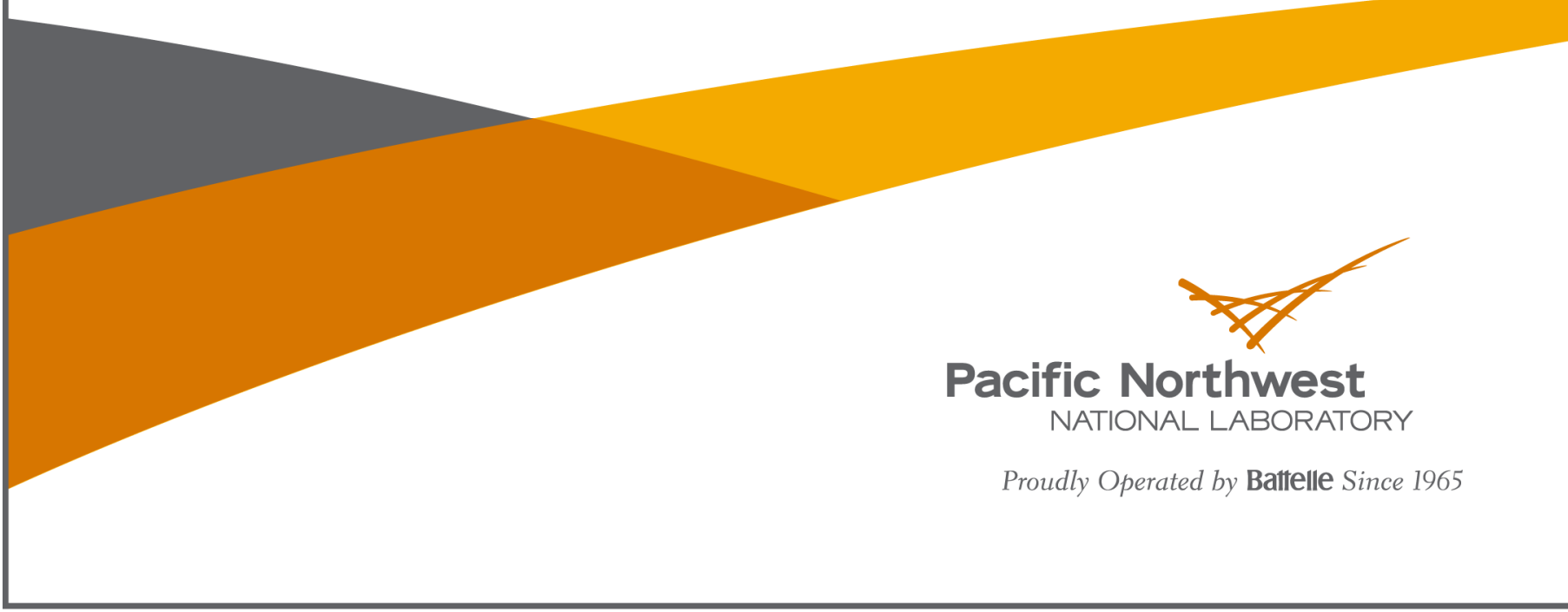




\title{
DISCLAIMER
}

This report was prepared as an account of work sponsored by an agency of the United States Government. Neither the United States Government nor any agency thereof, nor Battelle Memorial Institute, nor any of their employees, makes any warranty, express or implied, or assumes any legal liability or responsibility for the accuracy, completeness, or usefulness of any information, apparatus, product, or process disclosed, or represents that its use would not infringe privately owned rights. Reference herein to any specific commercial product, process, or service by trade name, trademark, manufacturer, or otherwise does not necessarily constitute or imply its endorsement, recommendation, or favoring by the United States Government or any agency thereof, or Battelle Memorial Institute. The vicws and opinions of authors expressed hercin do not necessarily state or reflect those of the United States Government or any agency thereof.

\author{
PACIFIC NOR'IHWES'I NATIONAL LABORA'TORY \\ operated by \\ Available to the public from the National Technical Information Service, \\ U.S. Department of Commerce, 5285 Port Royal Rd., Springfield, VA 22161 \\ ph: (800) 553-6847 \\ fax: $(703) 605-6900$ \\ email: orders@ntis.fedworld.gov \\ online ordering: http://www.ntis.gov/ordering.htm
}




\title{
Juvenile Salmon Acoustic Telemetry System Transmitter Downsize Assessment
}

\author{
TJ Carlson \\ MJ Myjak
}

April 2010

Prepared for the U.S. Army Corps of Engineers, Portland District under an Interagency Agreement with the U.S. Department of Energy Contract DE-AC05-76RL01830

Pacific Northwest National Laboratory

Richland, Washington 99352 


\section{Summary}

At the request of the U.S. Army Corps of Engineers, Portland District, researchers from Pacific Northwest National Laboratory investigated the use of an application-specific integrated circuit (ASIC) to reduce the weight and volume of Juvenile Salmon Acoustic Telemetry System (JSATS) transmitters while retaining current functionality. Review of the design of current JSATS transmitters identified components that could be replaced by an ASIC while retaining the function of the current transmitter and offering opportunities to extend function if desired. ASIC design alternatives were identified that could meet transmitter weight and volume targets of $200 \mathrm{mg}$ and $100 \mathrm{~mm}^{3}$. If alternatives to the cylindrical batteries used in current JSATS transmitters can be identified, it could be possible to implant ASIC-based JSATS transmitters by injection rather than surgery. Using criteria for the size of fish suitable for surgical implantation of current JSATS transmitters, it was concluded that fish as small as $70 \mathrm{~mm}$ in length could be implanted with an ASIC-based transmitter, particularly if implantation by injection became feasible. 


\section{Acknowledgments}

The authors thank Blaine Ebberts, U.S. Army Corps of Engineers (USACE), Portland District, for his patience waiting completion of this report and his assistance in identifying the biological targets for a downsized JSATS transmitter. We also thank Brad Eppard, USACE, Portland District, and Richard Brown, Pacific Northwest National Laboratory, for their review of the report. 


\section{Acronyms and Abbreviations}

$\begin{array}{ll}\text { ASIC } & \text { application-specific integrated circuit } \\ \text { ATS } & \text { Advanced Telemetry Systems, Inc., Isanti, Minnesota } \\ \text { DLL } & \text { delay-locked loop } \\ \text { FCRPS } & \text { Federal Columbia River Power System } \\ \text { g } & \text { gram(s) } \\ \text { JSATS } & \text { Juvenile Salmon Acoustic Telemetry System } \\ \text { kHz } & \text { kilohertz } \\ \text { LRP } & \text { log ratio of pressure change } \\ \text { mg } & \text { milligram(s) } \\ \text { mm } & \text { millimeter(s) } \\ \mu \text { F } & \text { microfarad(s) } \\ \text { nF } & \text { nanofarad(s) } \\ \text { nm } & \text { nanometer(s) } \\ \text { PIT } & \text { passive integrated transponder } \\ \text { PNNL } & \text { Pacific Northwest National Laboratory } \\ \text { PZT } & \text { piezoelectric transducer } \\ \text { RF } & \text { radio frequency } \\ \text { SC } & \text { Sonic Concepts, Inc., Bothell, Washington } \\ \text { USACE } & \text { U.S. Army Corps of Engineers } \\ \text { V } & \text { volt(s) }\end{array}$




\section{Contents}

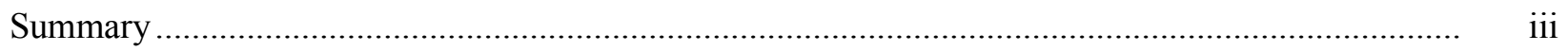

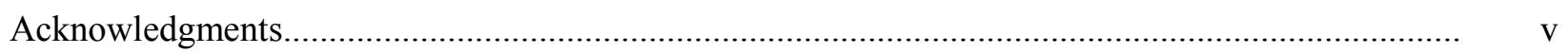

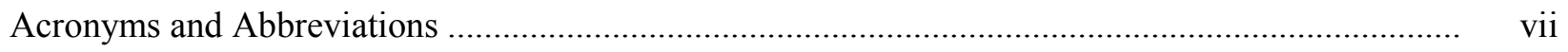

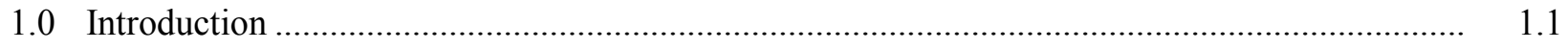

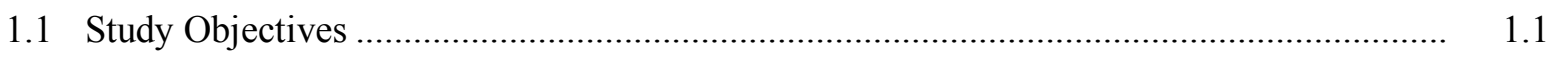

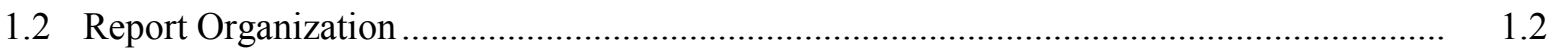

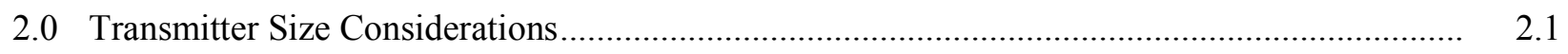

3.0 Downsize Alternatives Assessment .............................................................................

3.1 Application-Specific Integrated Circuit Overview..................................................... 3.2

3.1.1 Current JSATS Microtransmitter Functionality ................................................. 3.3

3.1.2 Application-Specific Integrated Circuit Packaging Alternatives ............................ 3.4

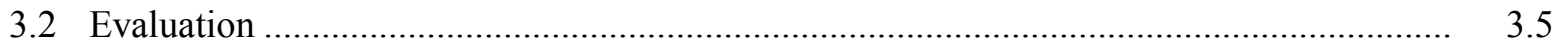

3.3 Potential Extended Microtransmitter Functionality ...................................................... $\quad 3.5$

3.4 Application-Specific Integrated Circuit Fabrication .................................................... 3.6

3.5 Application-Specific Integrated Circuit Design Alternatives........................................... 3.6

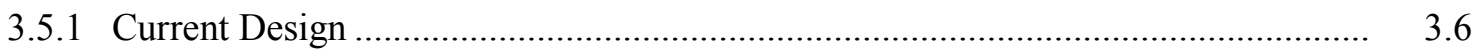

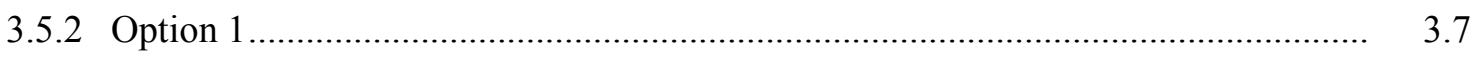

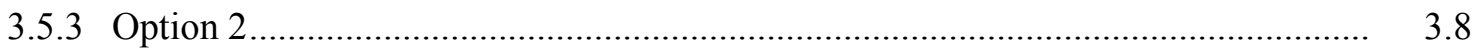

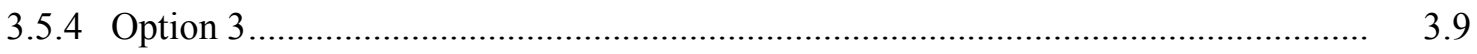

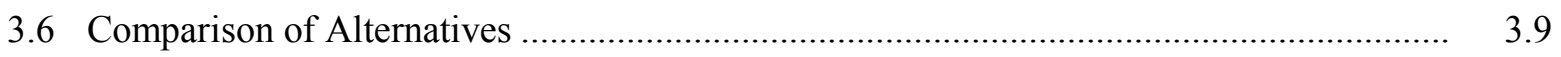

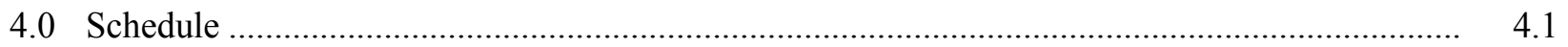

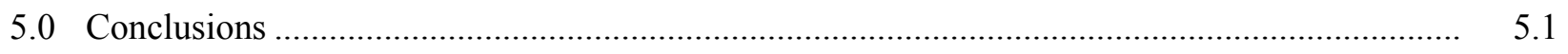

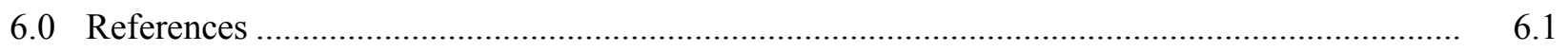




\section{Figures}

2.1 PIT tag (bottom) and JSATS dual-battery configuration acoustic transmitter (top) size comparison.

2.2 Mortal injury index for transmitter dry weights from $0 \mathrm{~g}$ to $0.536 \mathrm{~g}$ for fish weighing between $2 \mathrm{~g}$ and $30 \mathrm{~g}$

2.3 Mortal injury index for transmitter dry weights from $0 \mathrm{~g}$ to $0.536 \mathrm{~g}$ for fish between $60 \mathrm{~mm}$ and $150 \mathrm{~mm}$ in length

3.1 Reference design of current JSATS acoustic transmitter.

3.2 Option 1 design.....

3.3 Option 2 design....

\section{Tables}

2.1 Tagging alternatives used to investigate the effect of tag burden on the response of juvenile Chinook salmon to rapid decompression

3.1 Current and target properties of acoustic microtransmitters.

3.2 Options for integrating current JSATS microtransmitter functionality into an applicationspecific integrated circuit

3.3 Options for extending current functionality of an application-specific integrated circuit

3.4 Current MOSIS prices for fabricating a 2.25- $\mathrm{mm}^{2}$ unpackaged application-specific integrated circuit by technology alternatives

3.5 Size comparison of JSATS acoustic microtransmitters with those of application-specific integrated circuits

3.6 Weight comparison of acoustic microtransmitters.

4.1 Tasks and estimated duration for application-specific integrated circuit development 


\subsection{Introduction}

Acoustic telemetry has been identified as a technology for observation of behavior and assessment of survival for juvenile Chinook salmon passing through the Federal Columbia River Power System (FCRPS). Considerable effort has been expended to understand the biological effects of implantation of acoustic transmitters in yearling and subyearling Chinook salmon. Much additional effort has gone into development of autonomous and cabled receiving systems that can be deployed at dams and elsewhere in the river to detect fish bearing acoustic transmitters and process the resulting detection data to track the fish and provide data necessary to estimate survival by dam route of passage.

Bioeffects studies conducted to date show that reduction in the weight of Juvenile Salmon Acoustic Telemetry System (JSATS) transmitters will provide biological benefits and broaden the range of applications. Additional benefits, both biological and economic (reduction in costs of use), are possible through changes in the weight ${ }^{1}$, volume, and shape of a transmitter that would permit implantation by injection rather than surgery. In particular, biological benefits sought are

1. reduction in tag burden for subyearling Chinook salmon and similar-size salmonid migrants originating upstream of Bonneville Dam, to reduce the risk of bias in dam passage survival estimates resulting from differential probability of mortal injury between untagged and tagged fish when exposed to rapid decompression

2. reduction in tag burden for juvenile salmonids originating downstream of Bonneville Dam, to permit longer-term study of their behavior, habitat use, and survival during residence in the Columbia River estuary and the river reach between Bonneville Dam and the estuary

3. changes in the volume and shape of transmitters, to permit implantation by injection instead of surgical implantation.

The juvenile Chinook salmon and steelhead trout that originate upstream of Bonneville Dam are the main concern for improvements in dam passage at mainstem federal dams on the Snake and Columbia rivers but are not the only fish of concern in the Columbia River downstream of Bonneville Dam. Other juvenile Chinook salmon enter the lower river from several tributaries to the Columbia River. Many of the juvenile fish that enter the Columbia River downstream of Bonneville Dam exhibit a variety of behaviors that differ in significant ways from those that enter upstream of Bonneville Dam. They also differ in age and size. In addition, the river environment downstream of Bonneville Dam differs significantly from that upstream, particularly when the river becomes increasingly estuarine in contrast to the reservoir environments that dominate upstream of Bonneville. The combination of these differences makes it very difficult to directly apply currently available acoustic telemetry transmitters, receivers, and biological-effects knowledge gained in the mainstem Columbia River upstream of Bonneville Dam to fish in the lower reaches of the river and estuary.

\subsection{Study Objectives}

The work described in this report was undertaken at the request of the U.S. Army Corps of Engineers (USACE), Portland District, by Pacific Northwest National Laboratory (PNNL). This study is the first of

\footnotetext{
${ }^{1}$ References to weight in the text of this report are for the dry weight of fish and microtransmitters.
} 
four phases of a project to specify, design, prototype, and test a JSATS transmitter smaller in size than that currently in use. The primary objective of the study was to identify alternatives for decreasing the size of JSATS transmitters to achieve the biological effects noted above. Secondary objectives were to identify opportunities to reduce the manufacturing complexity and cost of JSATS transmitters and identify strategies to incorporate design alternatives into ongoing competitive procurement of JSATS transmitters.

\subsection{Report Organization}

In Section 2, we discuss the weight, volume, and shape targets for transmitter downsize and some of the expected biological benefits. In Section 3, we discuss identified transmitter downsize alternatives for implementation of a transmitter based on an application-specific integrated circuit (ASIC). Section 4 lists the tasks and schedule for development of an ASIC to enable transmitter downsize. In Section 5, conclusions drawn from the results of this study are presented. Published sources cited in the text are listed in Section 6. 


\subsection{Transmitter Size Considerations}

The passive integrated transponder (PIT) tag, a small passive radio frequency (RF) transponder (Figure 2.1), was introduced initially for use in juvenile Chinook salmon in the Columbia River. The PIT tag provided the means to tag large numbers of downstream migrants to obtain information about their migration through the FCRPS. To the present, because of its ease of implantation by injection, relatively limited biological effects, and its widespread acceptance for use in fish as small as $65 \mathrm{~mm}$ in length, the PIT tag has been the target in size and implantation method for telemetry devices.

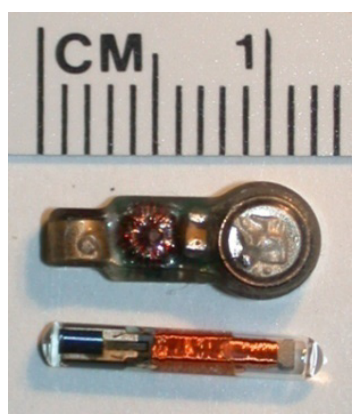

Figure 2.1. PIT tag (bottom) and JSATS dual-battery configuration acoustic transmitter (top) size comparison

Historically, the criterion for use of telemetry devices in fish has been in the form of the ratio of device dry weight to fish dry weight (called tag burden). Some biologists have suggested that this tag burden not exceed 2\% (Winter 1996). However, there have been numerous laboratory based studies that indicate that a tag burden higher than this may not influence swimming performance, growth, tag retention, or survival (Brown et al. 1999, 2006, 2010; Anglea et al. 2004; Zale et al. 2005). To this day, discussion continues about the $2 \%$ rule presented by Winter (1996). Jepsen et al. (2005) suggested that although it is "...important to minimize the size of a transmitter and disturbance to a fish," conclude that there is no "generally applicable rule" for tag burden (Jepsen et al. 2005). They assert that the maximum burden should be determined by study objective, tagging method, and the species/life stage to be studied. Those conducting research using acoustic telemetry in the FCRPS for the USACE have implicitly adopted this argument.

Figure 2.1 shows the differences in size and shape between the current JSATS transmitters and a PIT tag. The JSATS transmitter battery is one of the major tag elements that currently inhibit implantation of the tag by injection rather than surgery. Although not explicitly considered in this report, it is clear that any action taken to downsize JSATS transmitters must identify alternatives to replace the hearing aid batteries now used to power JSATS transmitters.

Beginning in 2006, the USACE initiated the use of rapid decompression as a stressor to evaluate the risk of injury to fish for telemetry devices by implantation method, burden, and various device characteristics. Initial studies developed the baseline response to rapid decompression for juvenile Chinook salmon that were not tagged. ${ }^{1}$. These studies developed the response of untagged fish expressed as the probability of mortal injury to rapid decompression for a range of acclimation pressures, nadir pressures, rate of change in pressure, and total dissolved gas pressure. The response of test fish to rapid

\footnotetext{
${ }^{1}$ TJ Carlson and RS Brown, Pacific Northwest National Laboratory, unpublished data.
} 
decompression was described using likelihood equations that related the exposure treatment variables to probability of mortal injury for subyearling and yearling Chinook salmon. Testing to assess the risk of mortal injury for treatments that include a range of acoustic transmitter designs and tag burdens is nearing conclusion.

The selection of rapid decompression as a stressor for evaluation of acceptable tag burdens is based on the need to evaluate potential bias in turbine and spill passage survival rate estimates made using data obtained from observations of dam passage of fish bearing telemetry devices and experiencing rapid decompression. In addition, exposure of tagged fish to rapid decompression provides a rapid assessment of the susceptibility of fish bearing telemetry devices to reduction in fitness compared to untagged fish. We use the results of recently completed studies on untagged juvenile Chinook salmon (Skalski and Seaburg 2010) and preliminary results of studies currently under way of tagged juvenile Chinook salmon ${ }^{2}$ to develop preliminary guidelines for the acceptable tag burden for fish less than $95 \mathrm{~mm}$ in length.

Research on the response of juvenile Chinook salmon varying in weight from approximately $7 \mathrm{~g}$ to 55 $\mathrm{g}$ (corresponds to fish length from approximately $90 \mathrm{~mm}$ to $165 \mathrm{~mm}$ ) to tag burdens over the range of less than $1 \%$ to a little over $6 \%$ of fish weight has been under way over the last two years and is nearing completion. The treatments (tag designs and combinations) in the study are shown in Table 2.2. All treatments were applied to fish over the weight range of approximately $9 \mathrm{~g}$ to $55 \mathrm{~g}$. Only the PIT tag treatment was applied to fish less than $9 \mathrm{~g}$ in weight. Statistical analysis of the response of fish to rapid decompression (Skalski and Seaburg 2010) indicated that the type of burden the test fish carried (i.e., PIT tag only through double-battery JSATS transmitter + PIT tag) was not a significant factor affecting fish response. The burden of the tag on the fish (tag weight divided by fish weight), in conjunction with variables describing features of the decompressive exposure, explained almost all of the variability in test results (Skalski and Seaburg 2010).

\footnotetext{
${ }^{2}$ TJ Carlson and RS Brown, Pacific Northwest National Laboratory, unpublished data.
} 
Table 2.1. Tagging alternatives used to investigate the effect of tag burden on the response of juvenile Chinook salmon to rapid decompression

\begin{tabular}{lcccc}
\hline $\begin{array}{l}\text { Transmitter / } \\
\text { tag treatment }\end{array}$ & Picture & $\begin{array}{c}\text { Weight in } \\
\text { Water (g) }\end{array}$ & $\begin{array}{c}\text { Weight in } \\
\text { Air (g) }\end{array}$ & Volume (mL) \\
\hline PIT & 0.059 & 0.096 & 0.036 \\
& 0.191 & 0.305 & 0.114 \\
Single battery & 0.250 & 0.400 & 0.150 \\
$\begin{array}{l}\text { Single battery } \\
\text { W/ PIT }\end{array}$ & & & \\
$\begin{array}{l}\text { Double battery } \\
\text { w/ PIT }\end{array}$ & 0.356 & 0.536 & 0.180 \\
\hline
\end{tabular}

The model that best fit the rapid decompression response data for both untagged and tagged juvenile Chinook salmon over the weight range inclusive of both subyearling and yearling run types is shown in Equation (2.1) (Skalski and Seaburg 2010).

$p_{\text {Mort }}($ hatchery fish $)=\frac{e^{-5.886+4.108 * \mathrm{LRP}+0.929 * \text { Tag Burden }}}{1+e^{-5.886+4.108 * \mathrm{LRP}+0.929 * \text { Tag Burden }}}$

where

Natural log ratio of pressure change (LRP) is defined as LRP $=\ln \left(\frac{\text { Acclimation pressure }}{\text { Nadir pressure }}\right)$.

Tag burden is defined as tag burden $=100 \times \frac{\text { tag.weight }}{\text { fish.weight }}$.

Equation (2.1) was used to estimate the response of juvenile Chinook salmon over the weight range from $2 \mathrm{~g}$ to $30 \mathrm{~g}$ (Figure 2.2), which corresponds to a length range of 60 to $150 \mathrm{~mm}$ (Figure 2.3). These estimates were made with the LRP set to zero. The response variable, mortal injury, refers to a subset of barotrauma injuries correlated with increased risk of mortality but is not a predictor of mortality (McKinstry et al. 2007). Here these predictions are used to indicate the potential biological benefits for reductions in the weight of JSATS transmitters. 


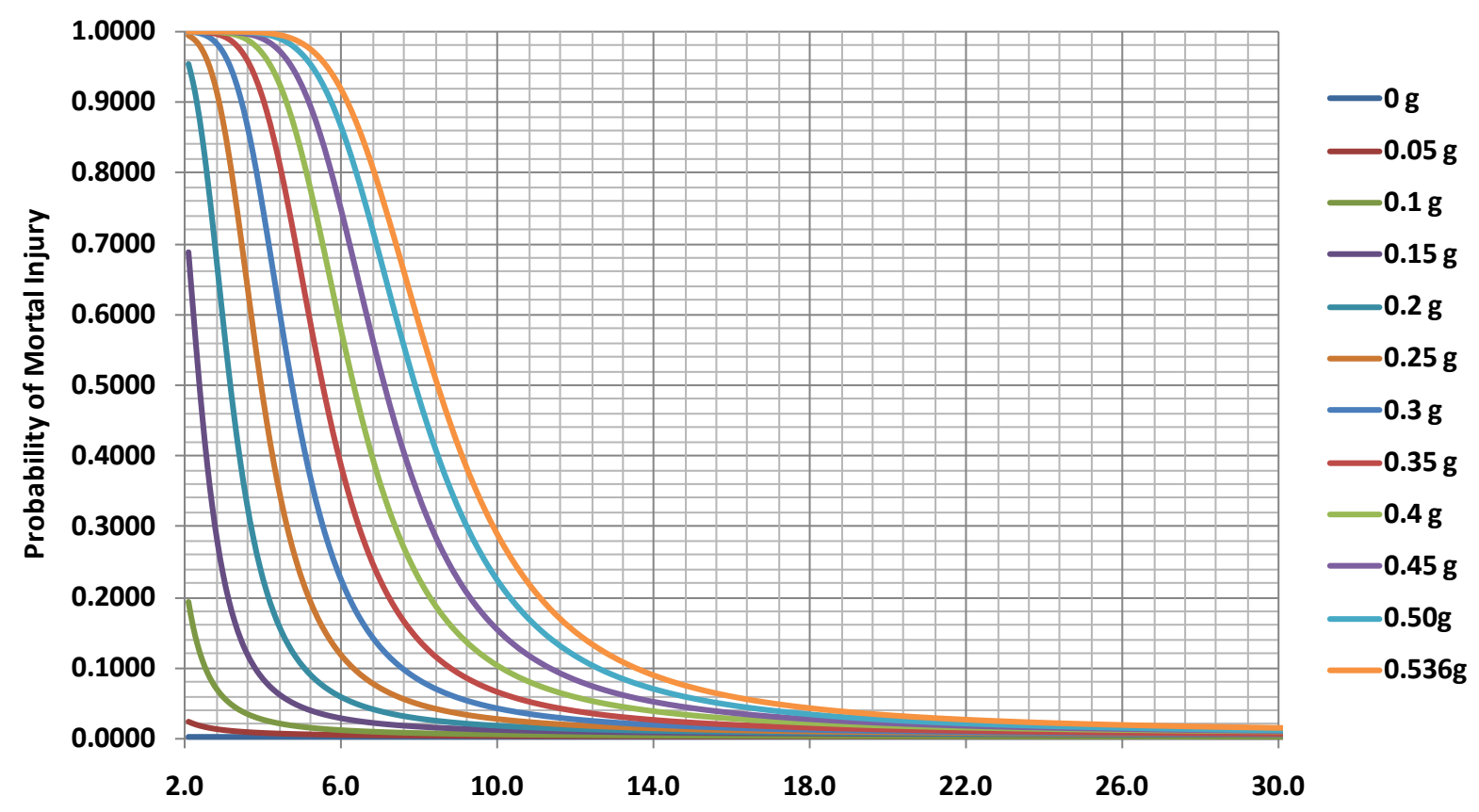

Figure 2.2. Mortal injury index for transmitter dry weights from $0 \mathrm{~g}$ to $0.536 \mathrm{~g}$ for fish weighing between $2 \mathrm{~g}$ and $30 \mathrm{~g}$

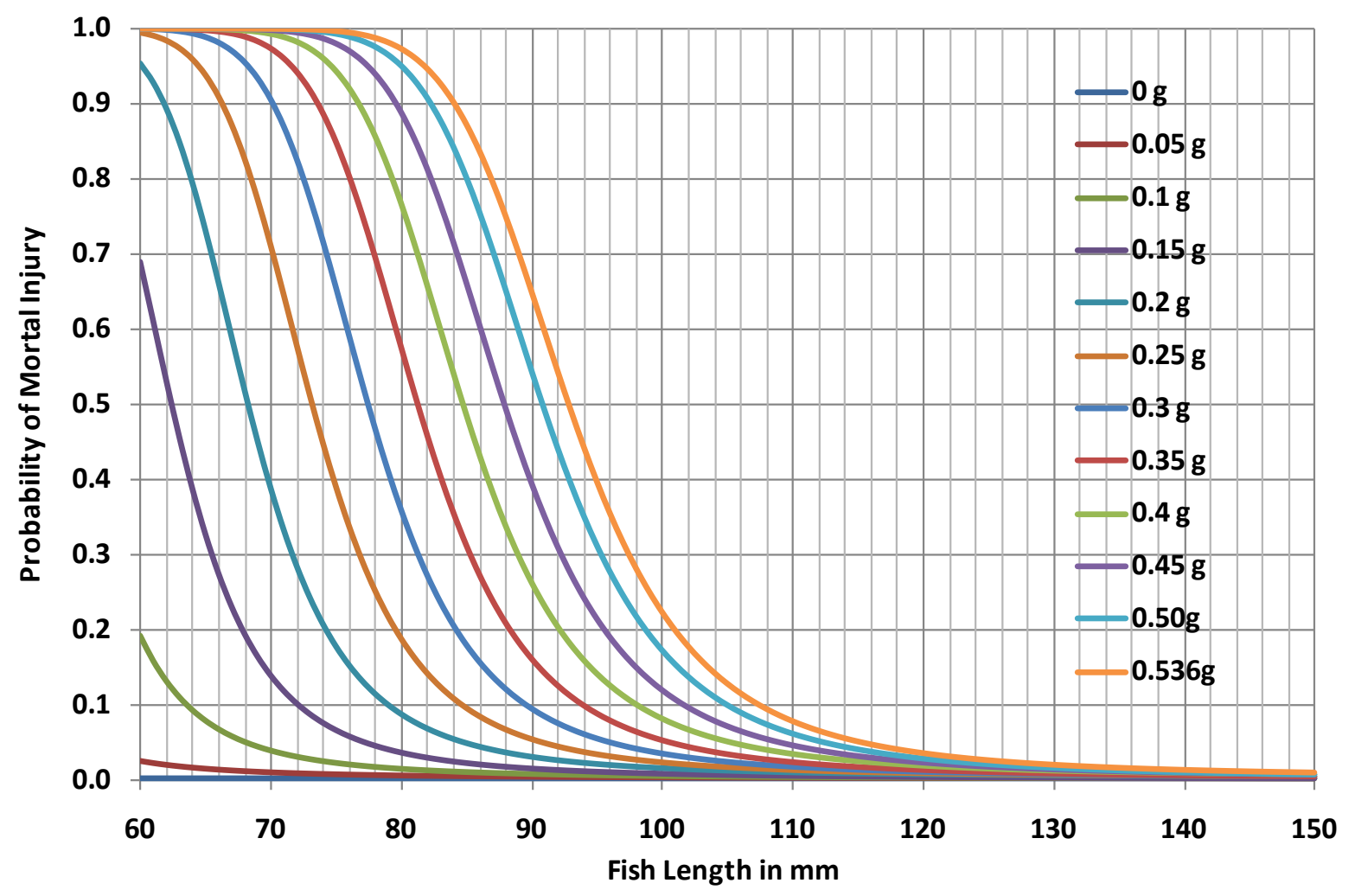

Figure 2.3. Mortal injury index for transmitter dry weights from $0 \mathrm{~g}$ to $0.536 \mathrm{~g}$ for fish between $60 \mathrm{~mm}$ and $150 \mathrm{~mm}$ in length 
At the present time, both JSATS and PIT tags are implanted in juvenile Chinook salmon greater than $95 \mathrm{~mm}$ in length (approximate weight of $9 \mathrm{~g}$ ) to estimate their survival and behavior while migrating through the mainstem FCRPS dams and estuary. The combined dry weight (weight in air) of the JSATS transmitter and PIT tag is $0.536 \mathrm{~g}$. For the smallest juvenile Chinook implanted, this is a burden of approximately $6 \%$. Inspection of Figures 2.2 and 2.3 indicates that this burden corresponds to a probability of mortal injury of 0.40 .

As indicated above, this value of mortal injury does not represent a prediction for probable mortality but is an indicator that can be used to assess the relative biological effect of a burden of this magnitude relative to increases and decreases in fish weight or length. For example, the relative biological effect of a burden of $0.536 \mathrm{~g}$ is roughly four times less for a juvenile Chinook salmon that weighs $14 \mathrm{~g} \mathrm{(108} \mathrm{mm}$ in length) than for a 9-g $(95-\mathrm{mm})$ juvenile Chinook at the lower threshold for tagging. These data also suggest that a reduction in tag weight to $0.200 \mathrm{~g}$ would reduce the relative biological effect for a juvenile Chinook at the current threshold for tagging to near that for an untagged or PIT-tagged juvenile Chinook. These data also indicate that, using the same criterion for the threshold on fish size for tagging, a tag weighing $0.2 \mathrm{~g}$ could be implanted in juvenile Chinook salmon approximately $3.5 \mathrm{~g}$ in weight or $70 \mathrm{~mm}$ in length. 


\subsection{Downsize Alternatives Assessment}

The primary goal in developing the JSATS was to create an open-source telemetry system for juvenile salmon that would meet USACE needs to estimate the survival of juvenile salmon migrating through the FCRPS and could be procured competitively by the government. Use of the current acoustic microtransmitter during several juvenile salmon outmigration seasons has demonstrated that its functionality is adequate to meet present USACE measurement objectives. Although its size meets current tag burden guidelines for most yearling Chinook salmon, reduction in size would reduce the possibility of adverse effects of implantation and would likely provide additional biological benefits for tagged fish. The current transmitter is too large for smaller juvenile Chinook salmon, particularly those found in the lower Columbia River and estuary that enter the river downstream of Bonneville Dam. Recent laboratory and in-field bioeffects studies indicate that juvenile Chinook less than $95 \mathrm{~mm}$ in length (approximately $9 \mathrm{~g}$ ) implanted with the current JSATS transmitter and PIT tag would have reduced survival and growth (Brown et al. 2010; Rub et al. 2010).

This section gives an assessment of how the current acoustic microtransmitter might be downsized to reduce biological stress to subyearling salmon and permit tagging of smaller juvenile Chinook salmon while retaining transmitter functionality. The main component of this effort is integrating a significant portion of the functionality onto an application-specific integrated circuit (ASIC). Not only would this approach reduce the size and weight of the tag, it also could increase the battery life and reduce manufacturing costs. In addition, it will become apparent in the following discussion that reductions in battery size and density are major factors affecting ability to achieve major reductions in microtransmitter weight and volume.

Table 3.1 lists the properties of past and current JSATS acoustic microtransmitters, along with the maximum acceptable JSATS microtransmitter target size and weight for this evaluation effort. Included are representative tags from Sonic Concepts (SC) and Advanced Telemetry Systems (ATS). A Digital Angel TX1400ST PIT tag is also included for comparison. From the analysis presented in Section 2, it is clear that reduction in transmitter weight beyond that of the target in Table 3.1 would be desirable.

Although the target does not include consideration of tag shape, any transmitter downsizing effort should focus also on alternatives to the batteries currently in use to move toward transmitter shapes that would permit implantation by injection.

Table 3.1. Current and target properties of acoustic microtransmitters

\begin{tabular}{lrrrrrr}
\hline Property & $2006 \mathrm{SC}$ & $2007 \mathrm{SC}$ & 2007 ATS & 2008 ATS & Digital Angel PIT & Target \\
\hline Length $(\mathrm{mm})$ & 16.10 & -- & -- & -- & 12.45 & 10.00 \\
Width $(\mathrm{mm})$ & 5.80 & -- & -- & -- & $\varnothing 2.01$ & $\varnothing 2.5$ \\
Height $(\mathrm{mm})$ & 4.07 & -- & -- & -- & & \\
Volume $\left(\mathrm{mm}^{3}\right)$ & 310 & 221 & 234 & 161 & 79 & 100 \\
Dry weight $(\mathrm{mg})$ & 640 & 601 & 606 & 485 & 107 & 200 \\
Wet weight $(\mathrm{mg})$ & 384 & 380 & 372 & 324 & 71 & 135 \\
\hline
\end{tabular}


In addition to reducing size and manufacturing costs of microtransmitters, the use of ASICs could integrate more functionality into the transmitters, extending the range of fish behavior, migration, ecology, and survival assessment applications.

\subsection{Application-Specific Integrated Circuit Overview}

The ASIC design process involves a number of steps:

- selection of an appropriate fabrication technology

- creation of circuit schematics

- simulation of the circuit schematics

- conversion of the schematics into a physical transistor layout

- design rule check of the layout

- back-conversion of the layout into a schematic with physical parameter values

- verification that the two schematics are functionally equivalent

- simulation of the back-converted circuit schematic

- transmission of the design to an appropriate foundry for fabrication.

These steps can be accelerated or automated with appropriate software tools. Commercial-quality tools are practically essential for designing an ASIC of any complexity. However, these tools are quite expensive and require specialized knowledge. Hence, the best option is to partner with a university or laboratory that has these resources.

The level of effort required to develop an ASIC depends mainly on the complexity of the design. However, purely digital circuits also require significantly less design time than mixed-signal circuits that integrate both analog and digital components. Mixed-signal design also requires more specialized expertise.

The total cost of an ASIC depends greatly on the design complexity and fabrication technology. Purely digital circuits require less design time than mixed-signal circuits that integrate both analog and digital components. The circuit complexity affects both the design time and the physical size of the layout. If the physical size is limited, a more expensive fabrication technology must be used.

The fabrication process incurs a large initial cost to create the necessary set of photolithography masks. These masks usually cost many thousands of dollars. For low-volume applications, services such as MOSIS ${ }^{1}$ can reduce this cost by combining designs from multiple customers onto the same silicon wafer. The individual dice are separated after fabrication. Each lot contains 40 parts. The prices would roughly scale with area. For higher-volume applications, MOSIS also offers dedicated runs, with one customer paying the total cost.

\footnotetext{
${ }^{1}$ http://www.mosis.com.
} 


\subsubsection{Current JSATS Microtransmitter Functionality}

A large portion of the current JSATS microtransmitter could be integrated onto a single ASIC. The small microcontroller currently performs all message timing and generates the output sequence for the drive transistors. One could easily replace this component with dedicated digital circuitry. The circuitry would need some nonvolatile memory for storing the unique tag address. Many fabrication technologies now support flash memory, so this requirement is not of concern. The digital logic could be designed to run at low voltage, potentially eliminating one of the batteries now required, or permitting the use of other batteries that are smaller in size than those currently being used.

In addition, the comparator and output drive transistors could be combined easily with the digital logic on the ASIC. Some fabrication technologies support two different transistor voltage levels, so that the digital logic could run at low voltage while the input and outputs could support a larger voltage range.

Integrating the analog components onto the ASIC would be more challenging. The passive resistors and capacitors may seem like an obvious choice, but the absolute accuracy of on-chip passive components is rather poor $( \pm 25 \%)$. In addition, capacitors in particular consume a large area: a general rule of thumb is $1 \mathrm{~mm}^{2} / \mathrm{nF}$. One upside of on-chip passive components is that the matching accuracy between two identical devices is very good $( \pm 0.1 \%)$. The differential resistors in the current JSATS microtransmitter might benefit from this accuracy, although the capacitors are probably too large. An alternative is to replace the resistors and capacitors in the output drive stage with a switched-capacitor filter. This analog circuit design technique uses small capacitors and transistor switches to produce the same overall functionality.

Another concept is to replace the external resonator with a temperature-compensated delay-locked loop (DLL). This circuit essentially contains a ring of digital inverters that oscillates at a predetermined frequency. The circuit could also be tunable in the field to compensate for process variations. With these techniques, the frequency error of the transmitted signal might decrease from $0.5 \%$ to $0.1 \%$, making the receiver design much less complex.

Finally, the wire-wound transformer in the current microtransmitter is an ideal candidate for replacement, due to its large size and expensive assembly process. The transformer is used to step up the current for the piezoelectric transducer (PZT) crystal. An alternative is to replace the transformer with a charge pump. This circuit uses a small, continuous current to gradually charge a series of capacitors. The voltage level of the output can be several times the voltage of the input. Preliminary calculations indicate that around four external $100-\mu \mathrm{F}$ capacitors would be required. Thus, this alternative might not reduce the area but would reduce the assembly cost.

Table 3.2 summarizes the options discussed so far for the ASIC. Included is the overall complexity, additional requirements, and estimated risk of each alternative. 
Table 3.2. Options for integrating current JSATS microtransmitter functionality into an applicationspecific integrated circuit

\begin{tabular}{lllll}
\hline Component & \multicolumn{1}{c}{ Replacement } & Complexity & \multicolumn{1}{c}{ Requirements } & \multicolumn{1}{c}{ Risk } \\
\hline Microcontroller & Digital logic & Medium & Flash memory for tag address & Low \\
Comparator & On-chip comparator & Low & Dual-voltage process desirable & Low \\
Output transistors & On-chip transistors & Low & Dual-voltage process desirable & Low \\
Resistors & On-chip resistors & Low & Tolerate poor absolute accuracy & High \\
Resistors and capacitors & Switched capacitor filter & Medium & Poly/poly capacitors desirable & Medium \\
Resonator & Delay locked loop & Medium & Flash memory for tuning & Medium \\
Transformer & Charge pump & Medium & External capacitors & Medium \\
\hline
\end{tabular}

\subsubsection{Application-Specific Integrated Circuit Packaging Alternatives}

Typically, integrated circuits are packaged within a black ceramic or plastic case after fabrication. The input and output pads on the die are wire-bonded to the package pins, which are then soldered to the underlying circuit board. An alternative is bare die assembly, whereby the unpackaged die is bonded directly to the circuit board. This approach not only reduces the physical size of the circuit but also improves performance and reliability. Using this approach for the ASIC in the acoustic microtransmitter would significantly reduce the size and weight. In fact, the approach might even be essential to meet the target requirements.

Several alternatives are available for mounting an unpackaged die to the circuit board:

- chip-on-board - The die is attached to the circuit board with epoxy, bottom-side down. A conductive epoxy may be used to provide an additional connection to ground. The input and output pads are wire-bonded to the circuit board. Finally, the die is covered with a protective coating that minimizes stresses on the wire bonds.

- flip-chip bumping - The die is attached to the circuit board, top-side down, such that the input and output pads make contact with corresponding pads on the circuit board. A special solder or adhesive ensures good electrical contact between the pads. A protective coating is then applied as a backfill and underfill for the die. This approach requires less area than chip-on-board and further improves the reliability.

- stacked die - This option is available only with custom integrated circuits that are designed to stack on top of each other.

A number of companies, such as Interconnect Systems Inc. ${ }^{2}$, offer bare die assembly services. Clearly, this process requires special equipment due to the handling requirements of unpackaged die. However, according to one distributor for bare die components ${ }^{3}$, bare die assembly actually saves money for high-volume applications, due to the increased functionality per square area and streamlined assembly

\footnotetext{
${ }^{2}$ http://www.isipkg.com.

3 http://www.ditech.co.uk/productsandservices/savingcost.htm.
} 
flow. For a production run of 1 million components, the unit cost might range around $\$ 0.41$ for chip-onboard and $\$ 0.20$ for solder flip-chip bumping, versus $\$ 0.78$ for standard packaging.

\subsection{Evaluation}

Using bare-die assembly would significantly reduce the size and weight of the microtransmitter. In all, the resistors, capacitors, comparator, and output transistors could be replaced with unpackaged parts with identical functionality. It might be necessary to change the microcontroller if the component is not provided as a bare die. As stated earlier, a custom ASIC could readily be used in unpackaged form. Parts that would not likely have an unpackaged equivalent include the activation switch, transformer, battery, and PZT crystal.

In general, bare-die assembly is becoming popular for high-volume designs with a custom ASIC, because the additional cost of equipment is offset by the lower complexity of fabrication. However, the cost would likely increase for this application, due to the additional cost of procuring components plus the special manufacturing requirements. The overall risk is low if the procurement and assembly are carried out by companies with experience in the area. Further study would be necessary to determine whether bare-die assembly would be a viable approach for the acoustic microtransmitter.

\subsection{Potential Extended Microtransmitter Functionality}

The ASIC can integrate other functionality beyond that provided by the current microtransmitter. For example, the integrated circuit could contain an amplifier that would enable the PZT crystal to be used as a receiver as well as a transmitter. This approach would enable a number of capabilities: placing individual tags in active or hibernation mode, synchronizing the transmit frequency to the frequency of the receiver, and storing location information on the tag through the use of position beacons. It is also possible for the tag to use an RF receiver, although this approach would require an external antenna.

Depending on the remaining area available, other transducers conceivably could be placed on the tag as well. A pressure transducer would provide an estimation of the depth, which substantially reduces the complexity of the tracking problem. A temperature sensor might indicate whether the fish was alive or dead. The ASIC would contain the amplifier or other circuitry required for the new sensors.

Table 3.3 summarizes the options for extending the functionality of the microtransmitter by integrating more functionality into the ASIC.

Table 3.3. Options for extending current functionality of an application-specific integrated circuit

\begin{tabular}{llll}
\hline Component & Complexity & \multicolumn{1}{c}{ Requirements } & Risk \\
\hline PZT receiver & High & Possibly additional components & High \\
RF receiver & High & External antenna & High \\
Amplifier for pressure sensor & Medium & Calibration & Medium \\
Amplifier for temperature sensor & Medium & Calibration & Medium \\
\hline
\end{tabular}




\subsection{Application-Specific Integrated Circuit Fabrication}

ASIC fabrication is performed at an integrated circuit foundry. The fabrication process incurs a large initial cost to create the necessary set of photolithography masks. These masks cost many thousands of dollars and thus are economical for only high-volume production. The fabrication technology significantly affects the cost as well: a die with 90 -nm feature sizes might pack 30 times more transistors in the same area but cost 20 times more than a die with 500-nm feature sizes.

Usually, initial prototypes of an ASIC are fabricated and tested before a high-volume production run is started. Services such as MOSIS ${ }^{4}$ reduce the effective fabrication cost by combining designs from multiple customers onto the same silicon wafer. The individual dice are separated after fabrication. Table 3.4 lists the current prices for fabricating a $2.25-\mathrm{mm}^{2}$ unpackaged ASIC in various technologies. Each lot contains 40 parts; a maximum of six lots can be ordered. Customers can work with MOSIS to aid the transition between the prototyping stage and higher-volume production.

Table 3.4. Current MOSIS prices for fabricating a $2.25-\mathrm{mm}^{2}$ unpackaged application-specific integrated circuit by technology alternatives

\begin{tabular}{lrrrrr}
\hline Parameter & AMI C5 & AMS C35 & TSMC CL025 & TSMC CL018 & IBM 9SF \\
\hline Operating voltages (V) & 5.0 & $3.3 / 5.0$ & $2.5 / 3.3$ & $1.3 / 3.3$ & 1.0 \\
Feature size (nm) & 500 & 350 & 250 & 180 & 90 \\
Price per lot & $\$ 6,500$ & $\$ 13,800$ & $\$ 23,000$ & $\$ 45,000$ & $\$ 125,000$ \\
\hline
\end{tabular}

\subsection{Application-Specific Integrated Circuit Design Alternatives}

We have identified three alternatives for integrating the functionality of the acoustic microtransmitter into an ASIC. Option 1 is a low-risk approach that offers no major technical challenges. Option 2 is a medium-risk approach that requires additional development time but integrates more components onto the ASIC. Finally, Option 3 is another medium-risk approach that incorporates new functionality into the ASIC. This section first describes the reference design for the acoustic microtransmitter and then presents the three design alternatives in detail.

\subsubsection{Current Design}

Figure 3.1 illustrates the reference design of the current JSATS acoustic microtransmitter. A small microcontroller generates the desired output signals for the PZT transducer, which converts a voltage into an acoustic signal. These signals are filtered by a resistor-capacitor network, amplified by driver transistors, and stepped up by a transformer. A separate oscillator circuit, consisting of an amplifier and some passive components, generates the $417-\mathrm{kHz}$ carrier frequency for the transmission. The microcontroller also requires a small resonator to generate its internal clock.

\footnotetext{
${ }^{4}$ http://www.mosis.com.
} 


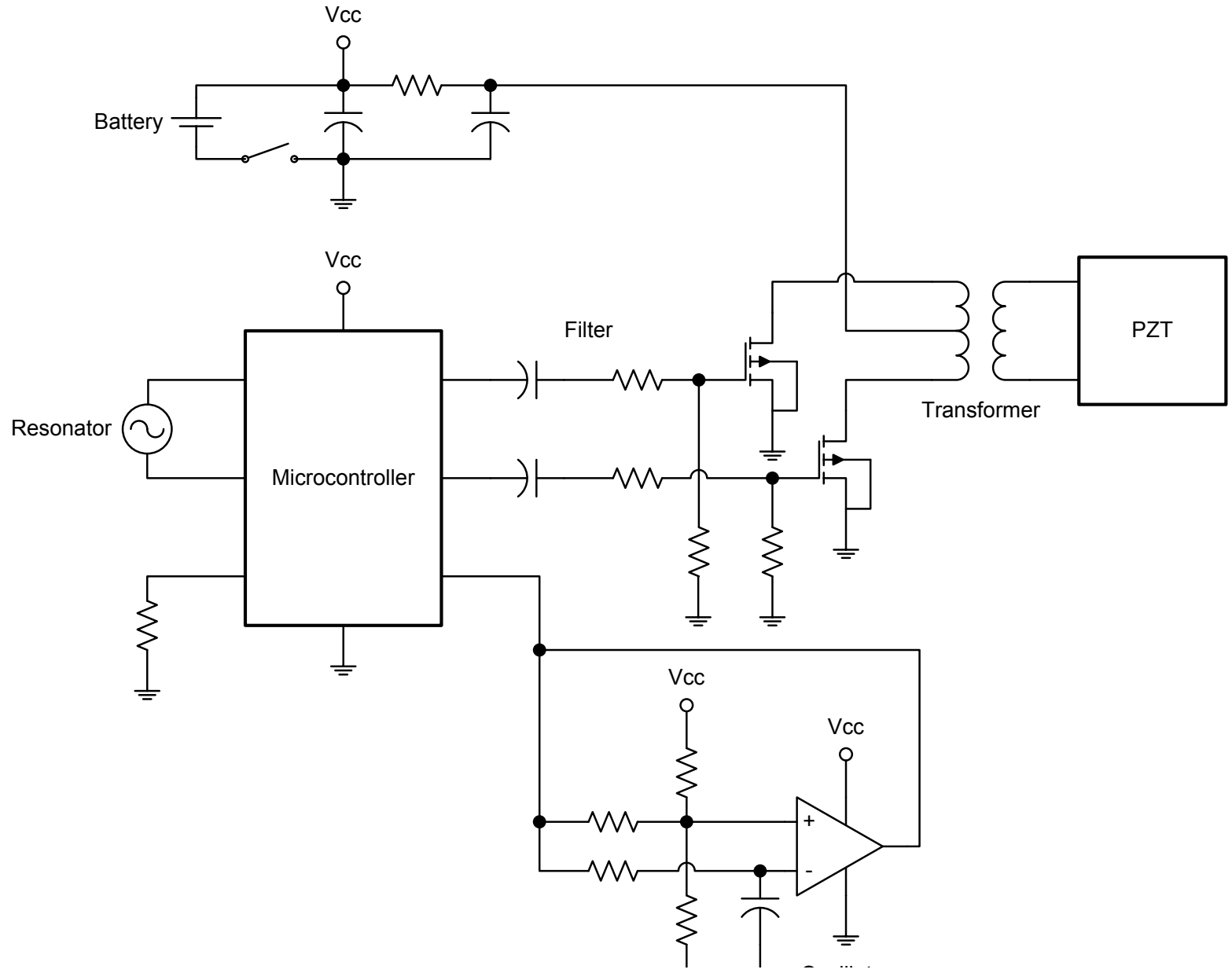

Figure 3.1. Reference design of current JSATS acoustic transmitter

Although this design has been successfully implemented on the $2006 \mathrm{SC}$ tag, it has a number of drawbacks. The microcontroller is underutilized, as it generates only a fixed waveform that transmits the specific tag code. The carrier frequency is susceptible to frequency error due to parameter variations in the oscillator circuit. Finally, the transformer has a high manufacturing cost due to its required size.

It should be noted that the ATS-manufactured tag uses a different design that achieves smaller area; however, detailed information about this design is not available.

\subsubsection{Option 1}

The first design alternative, shown in Figure 3.2, integrates the functionality of the microcontroller, oscillator, and driver transistors into an ASIC. The ASIC contains a small flash memory to store the tag code, a temperature-compensated phase-locked loop to generate the carrier frequency, and output transistors to drive the transformer. The passive filter is left unchanged. When a fixed number of carrier frequency cycles have elapsed, the ASIC drives the inputs of the passive filter with the tag code. The outputs of the passive filter are routed to the output transistors that drive the transformer. The tag code could be programmed into the ASIC after installation on the circuit board but prior to final conformal coating. 
With proper design of the phase-locked loop, the carrier frequency would have frequency accuracy superior to that of the current acoustic microtransmitters. The ASIC could store calibration coefficients in additional flash memory to compensate for parameter variations.

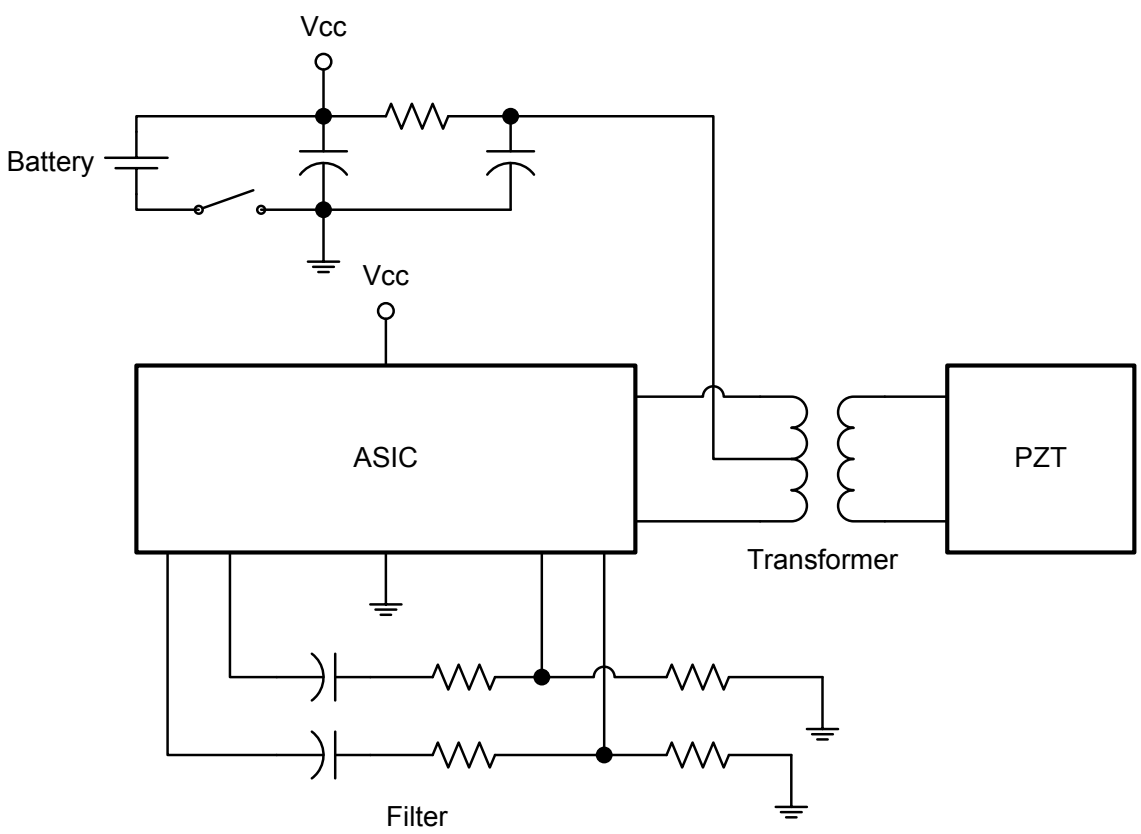

Figure 3.2. Option 1 design

The entire design would fit easily on a $2.25-\mathrm{mm}^{2}$ die in 500-nm fabrication technology or larger. Many technologies can support flash memory, so storing the tag code would be straightforward. One potential concern is that 500-nm technologies are usually designed for 5-V operation, whereas the battery would produce much lower voltage. This concern can be alleviated with low-power circuit design techniques. Approximately nine months of design time would be required to develop an initial version of the ASIC.

\subsubsection{Option 2}

Option 2, illustrated in Figure 3.3, further reduces the number of components required in the tag. The external filter is replaced by an internal switched-capacitor filter, with the capacitors implemented on the ASIC itself. In addition, the transformer is replaced with a switched inductor-capacitor circuit using an off-the-shelf inductor. The ASIC drives the PZT crystal directly with a 12-V output. The overall operation of the ASIC remains the same as that of option 1.

This approach is medium-risk due to a number of design challenges:

- design of a switched-capacitor filter with acceptable frequency stability

- design of a switched inductor-capacitor circuit with adequate drive capacity

- selection of an appropriate fabrication process that can drive the PZT transducer at $12 \mathrm{~V}$ and handle the necessary currents from the inductor. 
Because of these challenges, the ASIC would require approximately twelve months of design time and possibly a more expensive fabrication process.

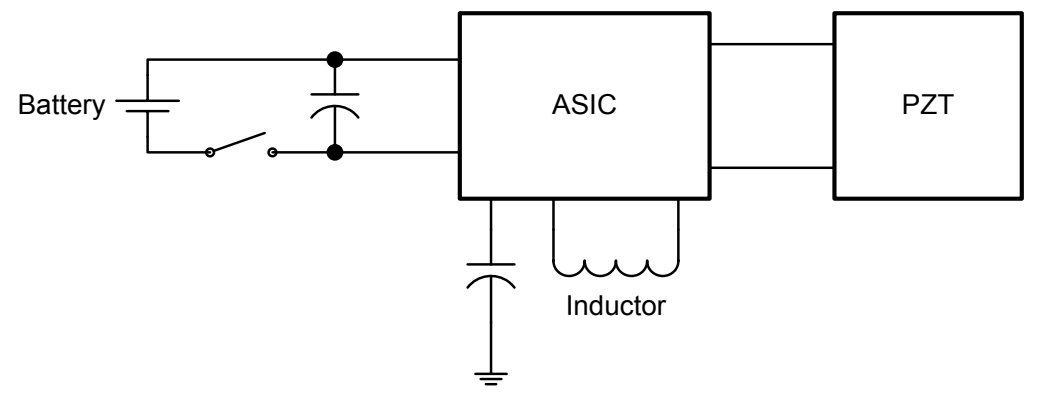

Figure 3.3. Option 2 design

\subsubsection{Option 3}

Option 3 uses the same circuit as option 2 but integrates additional functionality into the acoustic microtransmitter. For example, the ASIC could contain an amplifier that would enable the PZT crystal to be used as a receiver as well as a transmitter. This approach would enable a number of capabilities: placing individual tags in active or hibernation mode, synchronizing the transmit frequency to the frequency of the receiver, and storing location information on the tag through the use of position beacons.

Depending on the available area, other transducers conceivably could be placed on the tag as well. A pressure sensor would provide an estimation of the depth, which substantially reduces the complexity of tracking the fish. A temperature sensor might indicate whether the fish was healthy or dead. The ASIC would contain the amplifier or other circuitry required for the new sensors and would transmit the sensor data along with the tag code.

For this report, it is assumed that a small pressure sensor is added to the acoustic microtransmitter and that the ASIC contains the necessary interface circuitry. The development cost for option 3 would not be significantly greater than that for option 2 .

\subsection{Comparison of Alternatives}

To estimate the size and weight reduction that could be gained by using an ASIC, Tables 3.5 and 3.6 give a breakdown of the components required by the alternatives discussed previously. The known data from the 2006 SC tag and the 2008 ATS tag also are included. A tilde $(\sim)$ denotes an estimated value based on available data. Because a materials list for the ATS tag was not available, the volume and dry weight shown represent the values for a populated circuit board.

As shown, all three options have substantially reduced size and weight compared to the SC tag. Compared to the ATS tag, Option 1 has 17\% larger volume but 15\% lower weight. Option 2 has 5.6\% smaller volume and $23 \%$ lower weight. Finally, option 3 has approximately equal volume and $15 \%$ lower weight. From this analysis, it appears that options 2 and 3 show good promise.

Although using an ASIC will decrease the volume and weight of the current dual-battery JSATS transmitters, it is clear that a single battery implementation will be necessary to achieve the target volume 
of $100 \mathrm{~mm}^{3}$ and dry weight of $300 \mathrm{~g}$. Batteries with a different form factor than the current compressed cylinder will be needed before implantation by injection is feasible.

Table 3.5. Size comparison of JSATS acoustic microtransmitters with those of application-specific integrated circuits

\begin{tabular}{|c|c|c|c|c|c|c|c|c|c|c|}
\hline \multirow[b]{2}{*}{ Component } & \multicolumn{2}{|c|}{$2006 \mathrm{SC}$} & \multicolumn{2}{|c|}{2008 ATS } & \multicolumn{2}{|c|}{ Option 1} & \multicolumn{2}{|c|}{ Option 2} & \multicolumn{2}{|c|}{ Option 3} \\
\hline & No. & Volume $^{(a)}$ & No. & Volume & No. & Volume & No. & Volume & No. & Volume \\
\hline Resistor & 10 & 0.03 & & & 5 & 0.03 & 0 & & 0 & \\
\hline Capacitor (small) & 4 & 0.03 & & & 2 & 0.03 & 0 & & 0 & \\
\hline Capacitor (med) & 3 & 0.13 & & & 1 & 0.13 & 3 & 0.13 & 3 & 0.13 \\
\hline Capacitor (large) & 1 & 9.3 & & & 0 & & 0 & & 0 & \\
\hline Inductor & 0 & & & & 0 & & 1 & 0.13 & 1 & 0.13 \\
\hline Resonator & 1 & $\sim 2.9$ & & & 0 & & 0 & & 0 & \\
\hline ASIC & 0 & & & & 1 & 0.75 & 1 & 0.75 & 1 & 0.75 \\
\hline Microcontroller & 1 & 29 & & & 0 & & 0 & & 0 & \\
\hline Comparator & 1 & 5.8 & & & 0 & & 0 & & 0 & \\
\hline Driver & 1 & 6 & & & 0 & & 0 & & 0 & \\
\hline Circuit board & 1 & $\sim 5$ & & & 1 & $\sim 5$ & 1 & $\sim 5$ & 1 & $\sim 5$ \\
\hline Transformer & 1 & 36 & & & 1 & 36 & 0 & & 0 & \\
\hline PZT & 1 & 11.6 & 1 & 27 total & 1 & 11.6 & 1 & 11.6 & 1 & 11.6 \\
\hline Pressure sensor & 0 & & & & 0 & & 0 & & 1 & $\sim 9.3$ \\
\hline Battery $^{(b)}$ & 1 & 114 & 1 & 100 est. & 1 & 100 & 1 & 100 & 1 & 100 \\
\hline Coating & 1 & 90 & 1 & $\sim 34$ & 1 & $\sim 34$ & 1 & $\sim 34$ & 1 & $\sim 34$ \\
\hline Total 2 Battery & & 310 & & 161 & & 188 & & 152 & & 161 \\
\hline Total 1 Battery & & & & & & 138 & & 102 & & 111 \\
\hline
\end{tabular}

(a) All volumes in cubic millimeters $\left(\mathrm{mm}^{3}\right)$.

(b) Battery is composed of two batteries each with a volume of $50 \mathrm{~mm}^{3}$. 
Table 3.6. Weight comparison of acoustic microtransmitters

\begin{tabular}{|c|c|c|c|c|c|c|c|c|c|c|}
\hline \multirow[b]{2}{*}{ Component } & \multicolumn{2}{|c|}{$2006 \mathrm{SC}$} & \multicolumn{2}{|c|}{2008 ATS } & \multicolumn{2}{|c|}{ Option 1} & \multicolumn{2}{|c|}{ Option 2} & \multicolumn{2}{|c|}{ Option 3} \\
\hline & No. & Weight $^{(a)}$ & No. & Weight & No. & Weight & No. & Weight & No. & Weight \\
\hline Resistor & 10 & 0.14 & & & 5 & 0.14 & 0 & & 0 & \\
\hline Capacitor (small) & 4 & 0.28 & & & 2 & 0.28 & 0 & & 0 & \\
\hline Capacitor (med) & 3 & 1.2 & & & 1 & 1.2 & 3 & 1.2 & 3 & 1.2 \\
\hline Capacitor (large) & 1 & 32.4 & & & 0 & & 0 & & 0 & \\
\hline Inductor & 0 & & & & 0 & & 1 & 1.2 & 1 & 1.2 \\
\hline Resonator & 1 & $\sim 8.2$ & & & 0 & & 0 & & 0 & \\
\hline ASIC & 0 & & & & 1 & 2 & 1 & 2 & 1 & 2 \\
\hline Microcontroller & 1 & $\sim 82$ & & & 0 & & 0 & & 0 & \\
\hline Comparator & 1 & $\sim 16$ & & & 0 & & 0 & & 0 & \\
\hline Driver & 1 & $\sim 17$ & & & 0 & & 0 & & 0 & \\
\hline Circuit board & 1 & $\sim 10$ & 1 & 75 total & 1 & $\sim 10$ & 1 & $\sim 10$ & 1 & $\sim 10$ \\
\hline Transformer & 1 & 40 & 1 & 21 & 1 & 36 & 0 & & 0 & \\
\hline PZT & 1 & 36 & 1 & 30 & 1 & 11.6 & 1 & 11.6 & 1 & 11.6 \\
\hline Pressure sensor & 0 & & 0 & & 0 & & 0 & & 1 & $\sim 32$ \\
\hline Battery $^{(b)}$ & 1 & 270 & 1 & 270 & 1 & 270 & 1 & 270 & 1 & 270 \\
\hline Coating & 1 & 112 & 1 & $\sim 44$ & 1 & $\sim 44$ & 1 & $\sim 44$ & 1 & $\sim 44$ \\
\hline Total 2 Battery & & 630 & & 440 & & 376 & & 342 & & 374 \\
\hline Total 1 Battery & & & & & & 241 & & 207 & & 239 \\
\hline
\end{tabular}

(a) All weights in milligrams (mg).

(b) Battery is composed of two batteries, each with a weight of $135 \mathrm{mg}$. 


\subsection{Schedule}

The logical next step in the acoustic microtransmitter downsize project is the development of an ASIC. Table 4.1 lists the tasks and their expected duration for ASIC development. This analysis assumes that option 2 or option 3 would be chosen. Tasks 1 through 5 lead to the development of an initial prototype ASIC. A second version of the ASIC would be created and tested in tasks 6 through 8 to resolve any design issues.

Table 4.1. Tasks and estimated duration for application-specific integrated circuit development

\begin{tabular}{|c|c|c|}
\hline & Description & $\begin{array}{l}\text { Duration } \\
\text { (months) }\end{array}$ \\
\hline & $\begin{array}{l}\text { Specification } \\
\text { - } \quad \text { Definition of requirements } \\
\text { - Selection of appropriate fabrication process }\end{array}$ & 1 \\
\hline 2. & $\begin{array}{l}\text { Design } \\
\text { - } \quad \text { Creation of circuit schematics } \\
\text { - Simulation of circuit schematics to verify functionality }\end{array}$ & 3 \\
\hline 3. & $\begin{array}{l}\text { Layout } \\
\text { - } \quad \text { Conversion of schematics into transistor layout } \\
\text { - Design rule check of layout } \\
\text { - } \quad \text { Back-conversion of layout into schematic, with } \\
\text { - } \quad \text { parasitic capacitances and resistances } \\
\text { - Simulation of back-converted circuit schematic }\end{array}$ & 5 \\
\hline 4. & $\begin{array}{l}\text { Fabrication of Initial ASIC } \\
\text { - } \quad \text { Fabrication of initial integrated circuit } \\
\text { - } \quad \text { Packaging in standard case }\end{array}$ & 3 \\
\hline \multirow[t]{2}{*}{5.} & $\begin{array}{l}\text { Laboratory Testing } \\
\text { - } \quad \text { Verification of functionality } \\
\text { - } \quad \text { Assessment of performance in laboratory }\end{array}$ & 2 \\
\hline & Subtotal & 14 \\
\hline 6. & $\begin{array}{l}\text { Design Changes } \\
\text { - } \quad \text { Resolution of any issues with ASIC performance } \\
\text { - Selection of final package and assembly technique }\end{array}$ & 4 \\
\hline 7. & $\begin{array}{l}\text { Fabrication of Final ASIC } \\
\text { - } \quad \text { Fabrication of final integrated circuit } \\
\text { - } \quad \text { Assembly on miniaturized circuit board }\end{array}$ & 6 \\
\hline & Total & 24 \\
\hline
\end{tabular}




\subsection{Conclusions}

Based on this preliminary assessment of specifications, the following conclusions are offered:

1. Significant reductions in the volume and weight of JSATS microtransmitters are possible by using an ASIC to replace most of the components required for current designs. Further reductions are feasible by reducing the number of batteries needed from two to one.

2. Reduction in JSATS transmitter weight to $0.200 \mathrm{~g}$ would likely result in significant reduction in the biological effects of tagging and permit fish much smaller than the current threshold of tagging of 95-mm-long fish. Reduction in weight to this level is required to permit the use of acoustic telemetry to study the behavior and survival of the smaller juvenile Chinook salmon that enter the Columbia River downstream of Bonneville Dam.

3. Replacement of the batteries used in current JSATS transmitters by others lighter in weight with form factors that would permit transmitter shapes amenable to injection is highly desirable. Any effort to decrease the weight and volume of JSATS transmitters should include efforts to identify alternatives to the batteries currently used.

4. ASIC packaging and mounting alternatives offer opportunities to reduce the manufacturing cost of JSATS transmitters.

5. ASIC design alternatives could increase the functionality of the JSATS microtransmitter and thereby broaden its use for study of fish behavior and estimation of fish survival. 


\subsection{References}

Anglea SM, DR Geist, RS Brown, KA Deters, and RD McDonald. 2004. Evaluation of surgically implanted acoustic transmitters on the swimming performance and predator avoidance of juvenile Chinook salmon. North American Journal of Fisheries Management 24:162-170.

Brown RS, SJ Cooke, WG Anderson, and RS McKinley. 1999. Evidence to Challenge the " $2 \%$ Rule" for biotelemetry. North American Journal of Fisheries Management 19:867-871.

Brown RS, DR Geist, KA Deters, and A Grassell. 2006. Effects of surgically implanted acoustic transmitters greater than $2 \%$ of body mass on the swimming performance, survival, and growth of juvenile sockeye and fall Chinook salmon. Journal of Fish Biology 69:1626-1638.

Brown RS, RA Harnish, KM Carter, MW Boyd, KA Deters, and MB Eppard. 2010. An evaluation of the maximum tag burden for implantation of acoustic transmitters in juvenile Chinook salmon. North American Journal of Fisheries Management 30:499-505. DOI: 10.1577/M09-038.1.

Jepsen H, C Schreck, S Clements, and EB Thorstad. 2005. A brief discussion on the $2 \%$ tag/bodymass rule of thumb. In Aquatic Telemetry - Advances and Applications - Proceedings of the Fifth Conference on Fish Telemetry held in Europe Ustica, Italy, 9-13 June 2003, MT Spedicato, G Lembo, and G Marmulla (eds), pp. 255-259. Coispa Teconologia \& Ricerca and Food and Agriculture Organization of the United Nations, Rome, Italy.

McKinstry C, TJ Carlson, and RS Brown. 2007. Derivation of a Mortal Injury Metric for Studies of Rapid Decompression of Depth-Acclimated Physostomous Fish. PNNL-17080, Pacific Northwest National Laboratory, Richland, Washington.

Rub AMW, RS Brown, BP Sanford, KA Deters, LG Gilbreath, MS Meyers, ME Peterson, RA Harnish, EW Oldenburg, JA Carter, IW Welch, GA McMichael, JW Boyd, EE Hockersmith, and GM Matthews. 2010. Comparative Performance of Acoustic-Tagged and Passive Integrated Transponder-Tagged Juvenile Salmonids in the Columbia and Snake Rivers, 2007. U.S. Army Corps of Engineers, Portland District, Portland, Oregon.

Skalski JR and AG Seaburg. 2010. The Comprehensive Analysis of Mortal Injury for Hatchery Yearling and Subyearling Chinook Salmon Smolts Subject to Simulated Pressure Changes Associated with Turbine Passage. Columbia Basin Research, School of Aquatic and Fishery Sciences, University of Washington, Seattle.

Winter JD. 1996. Advances in underwater biotelemetry. In Fisheries Techniques, 2nd edition, BR Murphy and DW Willis (eds), pp. 555-590. American Fisheries Society, Bethesda, Maryland.

Zale AV, C Brooke, and WC Fraser. 2005. Effects of surgically implanted transmitter weights on growth and swimming stamina of small adult westslope cutthroat trout. Transactions of the American Fisheries Society 134:653-660. 


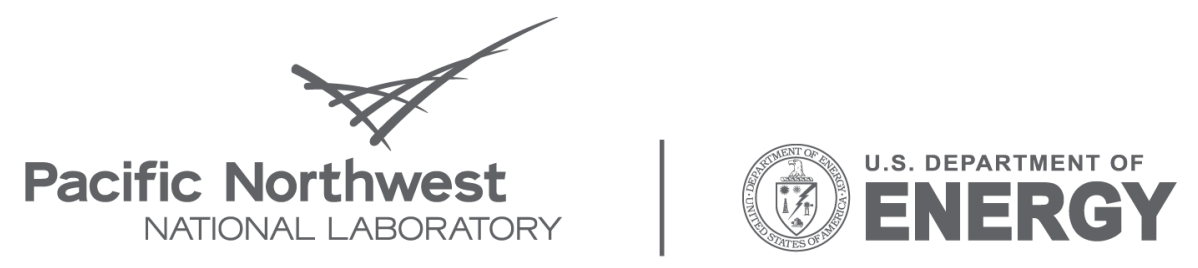

Proudly Operated by Battelle Since 1965

902 Battelle Boulevard

P.O. Box 999

Richland, WA 99352

1-888-375-PNNL (7665)

www.pnl.gov 